\title{
Cancer and Clinical Research
}

CASE REPORT

\section{Solitary Brain Metastasis in Low-Risk Prostate Cancer - A Case Report}

\author{
Sonha Nguyen ${ }^{1,2^{*}}$, Sameer Shah ${ }^{2}$, Neil Dudheker $^{3}$ and Vishal Ranpura ${ }^{4}$ \\ ${ }^{1}$ Department of Medicine, University of California Riverside, Riverside, CA, USA \\ ${ }^{2}$ University of California Riverside, Riverside, CA, USA \\ ${ }^{3}$ University of California, San Diego, San Diego, CA, USA \\ ${ }^{4}$ Department of Hematology/Oncology, Kaiser Permanente, Riverside, CA, USA
}

*Corresponding author: Sonha Nguyen, University of California, 900 University Ave, Riverside, CA, 92521, USA, Tel: (951)827-1012

\begin{abstract}
Adenocarcinoma of prostate is the most common cancer affecting men in United States. The most common sites of prostate cancer metastasis are to bone and pelvic lymph nodes. Brain metastases are rare and reported in less than $1 \%$ of prostate cancer patients. Among the patients with brain metastasis, most of them had concurrent systemic disease involvement, such as: bone, lymph node, liver and/ or lung metastasis [1,2]. We report a case of patient with low risk prostate cancer, stage IV B, with well controlled systemic disease and developed brain metastasis.
\end{abstract}

\section{Keywords}

Brain metastases, Metastatic cancer, Prostate cancer, Stereotactic radiosurgery, Radiotherapy

\section{Case Presentation}

An 83-year-old Caucasian male was initially diagnosed with prostate adenocarcinoma involving left base, left mid and left apex, T1C and Gleason score 6 $(3+3)$ in 2008, His Prostate-Specific Antigen (PSA) at time of diagnosis was $7.49 \mathrm{ng} / \mathrm{mL}$. The patient underwent prostatectomy followed by adjuvant radiotherapy (73.8 Gy in 41 fractions) in February 2009. He was monitored with serial PSA after radiation treatment. In April of 2017, his PSA increased to $17 \mathrm{ng} / \mathrm{mL}$. He underwent bone scan which showed metastatic disease to the frontal bone, multiple sternocostal joints and ribs. He was started on treatment with bicalutamide $50 \mathrm{mg}$ daily for 1 month and $22.5 \mathrm{mg}$ of Lupron IM. After that,
Lupron was given based on testosterone levels (if testosterone level $>50 \mathrm{ng} / \mathrm{dL}$, patient will get Lupron injection). Patient responded very well to the treatment and his PSA levels decreased to $0.2 \mathrm{ng} / \mathrm{mL}$. In middle of 2018, patient's PSA was slowly increasing and by November 2018, it was $2.4 \mathrm{ng} / \mathrm{mL}$. His testosterone levels were below $50 \mathrm{ng} / \mathrm{dL}$ and was diagnosed with metastatic castrate resistant prostate cancer (mCRPC). Scans showed sclerosis of bone metastasis without new bone involvement or lymph node metastasis. Treatment with enzalutamide was recommended but not started because of high out-of-pocket expenses; therefore, he was continued with Lupron.

In January 2019, he complained of low energy and increasing sleepiness, otherwise, no neurological deficits. The Magnetic Resonance Imaging (MRI) of his brain showed a solitary lesion of $4.4 \times 3.6 \times 4.8 \mathrm{~cm}$ in the right frontal cortex with surrounding edema and leftward midline shift (Figure 1A). Computed tomography (CT) scan of chest/abdomen/pelvis and bone scan showed stable sclerotic low volume metastatic disease in bones compared to his previous scans. Patient underwent right-sided craniotomy and resection of the mass. Final pathology was consistent with adenocarcinoma, prostate origin. His mental status and memory improved after surgery. Three months later, he had underwent stereotactic radiation therapy to the surgical cavity with $55.5 \mathrm{~Gy}$ in right frontal brain cavity. Repeated MRI of the brain showed removal of the tumor and effect 


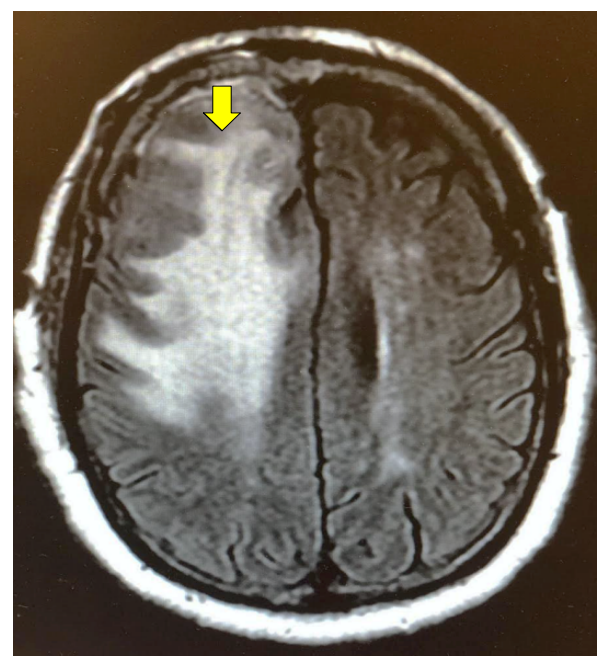

(A)

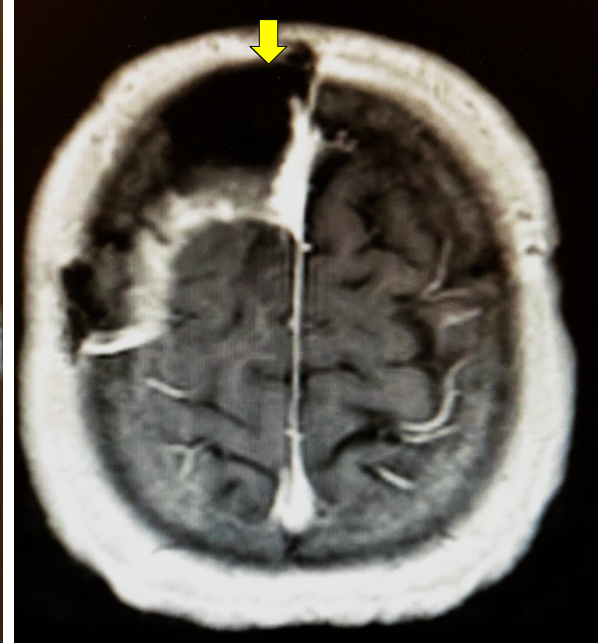

(B)

Figure 1: Magnetic Resonance Imaging of the brain at the axial plane. A) The hyperintense area (yellow arrow) showed lobulated, heterogeneously enhancing mass of $4.4 \times 3.6 \times 4.8 \mathrm{~cm}$ within the right frontal lobe with surrounding edema, effecament of the right frontal horn, and a $1.6 \mathrm{~cm}$ leftward midline shift, B) The T1 black hole (yellow arrow) showed removal of the tumor and effect of postoperative stereotactic radiation.

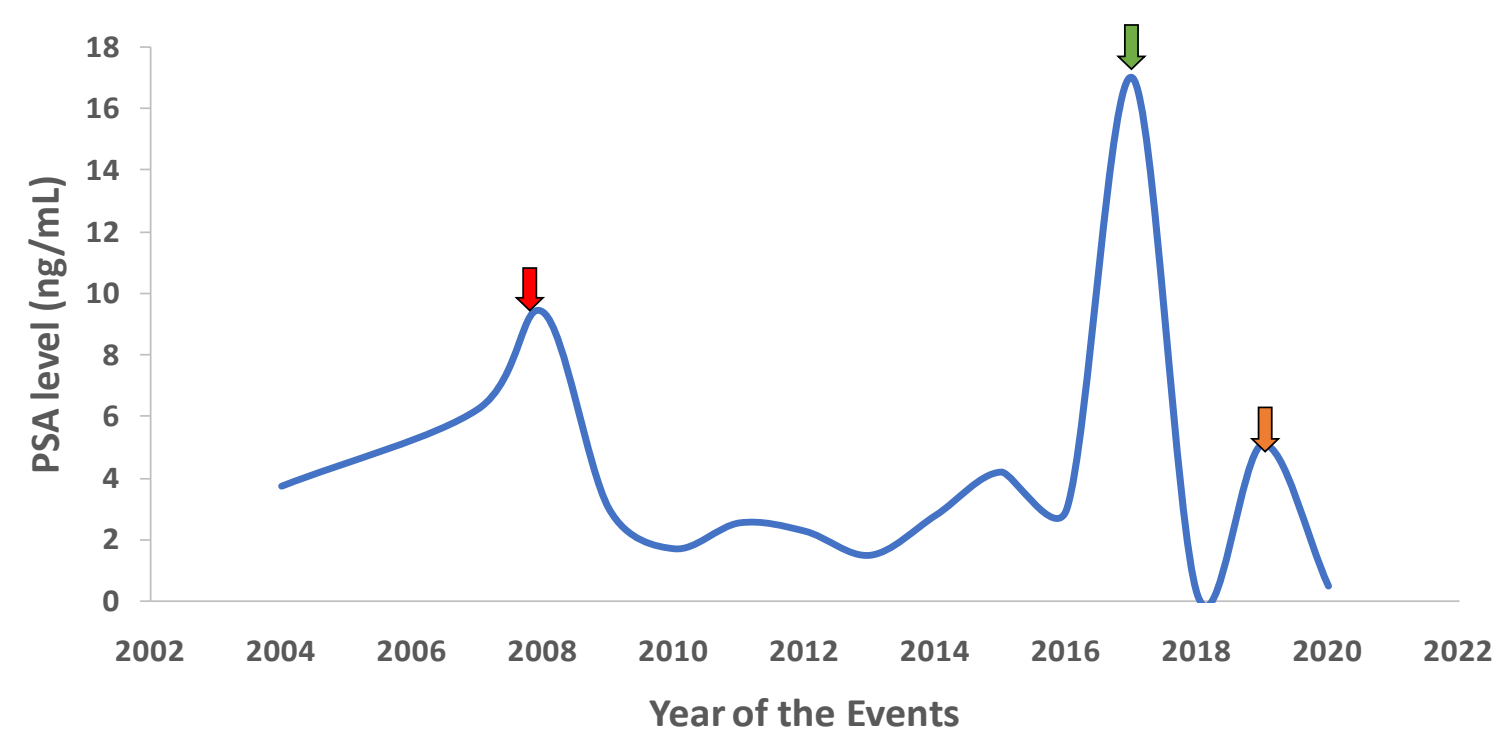

Figure 2: Serum level of PSA (ng/mL) since initial diagnosis of prostate cancer to presence. Red arrow: Elevation of PSA at initial diagnosis of prostate cancer followed by prostatectomy and radiotherapy. Green arrow: Bony metastasis and subsequently treated with bicalutamide and Lupron. Orange arrow: Diagnosis of brain metastasis followed by tumor resection and stereotactic radiation. PSA (Prostate-Specific Antigen).

of postoperative stereotactic radiation (Figure 1B). Patient's testosterone levels remained castrate and did not require further Lupron treatment.

The patient felt general decline in his performance status and wanted to focus on quality of life. He was referred to hospice in September 2019. In March 2020, patient was feeling better, he rescinded hospice and presented to oncology clinic for follow-up. His PSA level was $0.5 \mathrm{ng} / \mathrm{mL}$ (Figure 2) with no evidence of systemic progression on CT and bone scans. Figure 3 illustrated the timeline of the events since his initial diagnosis of prostate cancer.

\section{Discussion}

Metastatic brain lesions account for more than $50 \%$ of all central nervous system (CNS) tumors $[3,4]$. The median survival of patients with metastatic brain lesions is approximately 7-16 months from diagnosis [5]. While cutaneous melanoma and carcinomas of lung, breast, kidney, and colon commonly metastasize in the brain [6,7]; brain metastases from prostate cancer is very infrequent and poorly understood. Less than $1 \%$ of patients with prostate cancer were reported to have brain metastasis [8]. Among that, about five percent of the cases were diagnosed in postmortem examination [9]. Its rarity can be explained by earlier detection, indolent nature and effective treatments, preventing extraprostatic spread. Others possible explanation is that brain parenchyma is resistant to the establishment of metastatic foci by prostate adenocarcinoma cells [10]. 


\section{8-2009}

- Diagnosed with stage T1c adenocarcinoma of prostate from biopsy

-PSA: $9.38 \mathrm{ng} / \mathrm{dL}$; Gleason score 3+3

- Underwent prostatectomy and radiotherapy 7380 cGy in 41 fractions

2017
• PSA increased to $17 \mathrm{ng} / \mathrm{mL}$ and bone scan showed multiple bone metastases
• Continued with bicalutamide $50 \mathrm{mg}$ daily for 1 month and Lupron $22.5 \mathrm{mg}$
intramuscularly every 3 months

\section{8}

- PSA gradually increased to $5.1 \mathrm{ng} / \mathrm{mL}$ with testosterone levels down to $7 \mathrm{ng} / \mathrm{dl}$

- Diagnosed with metastatic castrate resistant prostate cancer (mCRPC)

-Patient could not afford Enzalutamide; therefore, he was continued on Lupron

\section{9}

-PSA arose from 0.4 to $3.7 \mathrm{ng} / \mathrm{dL}$ within 6 months prior

- MRI of the brain showed enhancing mass in right frontal lobe

- Underwent right frontal craniotomy and gross total resection with postoperative stereosurgery radiation $55.5 \mathrm{~Gy}$ at surgical bed

- Biopsy confirmed metastatic prostate adenocarcinoma

- Lupron was discontinued; patient wad referred to hospice

\section{0}

- Clinical presentation improved significantly with minimal residual neurological deficit

- Rescinded hospice

- The PSA level remained undetectable after brain lesion resection

Figure 3: Timeline of the events versus years since initial diagnosis of prostate cancer. PSA (Prostate-Specific Antigen), MRI (Magnetic Resonance Imaging).

Interestingly, the rate of brain metastasis from prostate cancer has been increasing over time. It is likely due to increasing availability of screening technique and the advanced treatment options, which lead to the longer lifespan of prostate cancer patients [11]. It may be also possible that a compromised immune system or a breakdown of the blood-brain barrier may be playing a role in brain metastasis as late event. In a study by Lynes, et al. study which published in 1986, the most common intracranial sites of prostate cancer metastasis were in leptomeninges up to $67.1 \%$; while cerebrum and cerebellum were less common, which took up to $25 \%$ and $8 \%$, respectively [1]. However, a recent study included ten prostate cancer patients with brain metastasis and showed metastasis is more common in intraparenchymal vs. dural metastasis, $60 \%$ vs. $20 \%$, respectively [12] It is arguable that the former study was limited before availability of MRI. Nonetheless, in our patient, brain metastasis was found in the cerebrum.

Neurological manifestations of brain metastasis were non-focal and could be asymptomatic [13], and often presented with cognitive changes and generalized headache. In our case, the patient presented with minimal neurological symptoms with generalized fatigue and sleepiness as clinical manifestations of brain metastasis eleven years after diagnosis of prostate cancer. MRI has been the gold standard to evaluate brain tumors. However, it remains a challenge to distinguish the lesions of brain metastasis from prostate cancer vs. from other types of cancers. It is due to its high variety of imaging presentation with no specific characteristics [2].

While surgical intervention has been playing a role in palliative treatment for patients with brain metastasis [14]; it is uncommonly performed due to the presence of widespread, systemic disease, low performance status, and short life expectancy. However, it has more benefit in patients with accessible, single brain lesion with good functional status. Meanwhile, stereotactic radiosurgery (SRS) is an established non-invasive ablative 
therapy widely adopted for unresected brain metastasis or as adjuvant therapy. In previous years, adjuvant Whole Brain Radiation Therapy (WBRT) along with surgical resection or with SRS has shown to significantly reduce the risk of recurrence from $60 \%$ to approximately $30 \%$ [15]. Hence, the combination of surgery and WBRT has been the standard of care for solitary brain metastasis. Despite, the concern of long-term neurotoxicity and quality of life limit the use of adjuvant WBRT [16]. Therefore, the use of high dose radiation to postoperative surgical bed with SRS is common in clinical practice [17], which was shown to have $79 \%$ local control rate at 12 months compared to $54 \%$ with observation only vs. with WBRT (80-90\%) [18]. In our patient, surgery and postoperative SRS to tumor bed were given and resulted in favorable outcome with good neurocognitive function thereafter.

The prognosis of prostate cancer patients with brain metastasis is very poor with reported median survival time ranging from 1 to 7.7 months $[19,20]$. It could be due to the majority of prostate cancer with brain metastasis also had concurrent distant metastasis [1]. In addition, the studied patients were high risk group with Gleason score $\geq 7$. Despite, there are some published case reports indicated better prognosis with more than 2 years survival after treatment [21,22]. Simultaneously, our patient had low-risk prostate cancer who is doing well after 16 months since diagnosis and treatment of brain metastasis and rescinded hospice. It can likely be explained by his well-controlled systemic disease and solitary brain metastasis which was optimally treated with no recurrence.

\section{Conclusion}

Prostate cancer with metastasis to brain is very rare, associated with widespread systemic disease and low median survival. We report a case of low-risk prostate cancer and well-controlled systemic disease presenting with solitary brain metastasis eleven years later, which emphasizes the importance of continued surveillance even in low-risk patients. Moreover, aggressive treatment of the metastatic site with tumor resection and adjuvant stereotactic radiation to the surgical bed deemed optimal in the management of this patient and associated with better outcome.

\section{Acknowledgement}

Data collection was approved by Kaiser Permanente, Riverside Human Research Protection Program, and informed written consent was obtained from the patient.

\section{Disclosures}

All authors stated that they had no conflicts of interest.

\section{Source of Support}

Not applicable.

\section{Author's Contribution}

$\mathrm{S}$ Nguyen drafted the manuscript. S Shah and $\mathrm{N}$ Dudheker assisted in literature review and drafting the manuscript. V Ranpura supervised and contributed to manuscript drafting. All authors critically reviewed the manuscript and approved its final version.

\section{References}

1. Lynes W, Bostwick DG, Freiha F, Stamey T (1986) Parenchymal brain metastases from adenocarcinoma of the prostate. Urology 28: 280-287.

2. Vaios Hatzoglou, Gita V Patel, Michael J Morris, Kristen Curtis, Zhigang Zhang, et al. (2014) Brain Metastases from Prostate Cancer: An 11-Year Analysis in the MRI Era with Emphasis on Imaging Characteristics, Incidence, and Prognosis. J Neuroimaging 24: 161-166.

3. George Al-Shamy, Raymond Sawaya (2009) Management of brain metastases: The indispensable role of surgery. $J$ Neurooncol 92: 275-282.

4. Emmanouil Fokas, Joachim $P$ Steinbach, Claus Rödel (2013) Biology of brain metastases and novel targeted therapies: Time to translate the research. Biochim Biophys Acta 1835: 61-75.

5. Patricia S Steeg, Kevin A Camphausen, Quentin R Smith (2011) Brain metastases as preventive and therapeutic targets. Nat Rev Cancer 11: 352-363.

6. Anna C Obenauf, Joan Massagué (2015) Surviving at a distance: Organ-specific metastasis. Trends Cancer 1: 76-91.

7. Ann F Chambers, Alan C Groom, Ian C MacDonald (2002) Dissemination and growth of cancer cells in metastatic sites. Nat Rev Cancer 2: 563-572.

8. Saitoh $\mathrm{H}$, Hida M, Shimbo T, Nakamura K, Yamagata J, et al. (1984) Metastatic patterns of prostatic cancer. Correlation between sites and number of organs involved. Cancer 54: 3078-3084.

9. Demierre B, Berney J (1988) Intracranial metastasis of prostatic adenocarcinoma. J Neurosurgery 69: 644-646.

10. Tremont-Lukats IW, Bobustuc G, Lagos GK, Lolas K, Kyritsis AP, et al. (2001) Brain metastasis from prostate carcinoma: the MD Anderson Cancer Center experience. Cancer 98: 363-368

11. Ahmedin Jemal, Rebecca Siegel, Jiaquan Xu, Elizabeth Ward (2010) Cancer statistics, 2010. CA Cancer J Clin 60: 277-300.

12. Kanyılmaz G, Aktan M, Yavuz BB, Koç M (2019) Brain metastases from prostate cancer: A single-center experience. Turk J Urol 45: 279-283.

13. Posner JB (1996) Brain metastases: 1995. A brief review. J Neurooncol 27: 287-293.

14. Sawaya $R$ (2001) Considerations in the diagnosis and management of brain metastases. Oncology (Huntington) 15: 1157-1158.

15. Martin Kocher, Riccardo Soffietti, Ufuk Abacioglu, Salvador Villà, Francois Fauchon, et al. (2011) Adjuvant whole- brain radiotherapy versus observation after radiosurgery or surgical resection of one to three cerebral metastases: results of the EORTC 22952-26001 study. J Clin Oncol 29: 134-141.

16. PK Sneed, KR Lamborn, JM Forstner, MW McDermott, S Chang, et al. (1999) Radiosurgery for brain metastases: Is whole brain irradiation necessary? Int $\mathrm{J}$ Radiat Oncol Biol Phys 43: 549-558. 
17. Soliman H, Das S, Larson DA, Sahgal A (2016) Stereotactic radiosurgery (SRS) in the modern management of patients with brain metastases. Oncotarget 7: 12318-12330.

18. Scott G Soltys, John R Adler, John D Lipani, Paul S Jackson, Clara YH Choi, et al. (2008) Stereotactic radiosurgery of the postoperative resection cavity for brain metastases. Int J Radiat Oncol Biol Phys 70: 187-193.

19. Caffo O, Veccia A, Fellin G, Mussari S, Russo L, et al. (2013) Frequency of brain metastases from prostate cancer: an 18-year single-institution experience. J Neurooncol 111: $163-167$.
20. Caffo O, Veccia A, Russo L, Galligioni E (2012) Brain metastases form prostate cancer: An emerging clinical problem with implications for the future therapeutic scenario. Future Oncol 8: 1585-1595.

21. Mithal P, Gong Y, Sirkis H, Aronowitz (2014) A brain lesion as the sole metastasis of prostate cancer. J Clin Urol.

22. Rao KG (1982) Carcinoma of prostate presenting as intracranial tumor with multiple cranial nerve palsies. Urology 19: 433-435. 